TRANSACTIONS OF THE

AMERICAN MATHEMATICAL SOCIETY

Volume 361, Number 5, May 2009, Pages 2305-2317

S 0002-9947(08)04806-X

Article electronically published on December 11, 2008

\title{
THE NEAR COHERENCE OF FILTERS PRINCIPLE DOES NOT IMPLY THE FILTER DICHOTOMY PRINCIPLE
}

\author{
HEIKE MILDENBERGER AND SAHARON SHELAH
}

Dedicated to Andreas Blass on the occasion of his 60th birthday.

\begin{abstract}
We show that there is a forcing extension in which any two ultrafilters on $\omega$ are nearly coherent and there is a non-meagre filter that is not nearly ultra. This answers Blass' longstanding question (1989) of whether the principle of near coherence of filters is strictly weaker than the filter dichotomy principle.
\end{abstract}

\section{INTRODUCTION}

By a filter we mean a proper filter on $\omega$. We call a filter non-principal if it contains all cofinite sets. Let $\mathscr{F}$ be a non-principal filter on $\omega$ and let $f: \omega \rightarrow \omega$ be finite-to-one (that means that the preimage of each natural number is finite). Then also $f(\mathscr{F})=\left\{X: f^{-1}(X) \in \mathscr{F}\right\}$ is a non-principal filter. Two filters $\mathscr{F}$ and $\mathscr{G}$ are nearly coherent if there is some finite-to-one $f: \omega \rightarrow \omega$ such that $f(\mathscr{F}) \cup f(\mathscr{G})$ generates a filter. We also say to this situation that $f(\mathscr{F})$ and $f(\mathscr{G})$ are coherent.

The filter dichotomy principle, FD, says that for every filter there is a finite-toone function $g$ such that $g(\mathscr{F})$ is either the filter of cofinite sets (also called the Fréchet filter) or an ultrafilter. In the latter case we call $\mathscr{F}$ nearly ultra. Talagrand 19 showed that there is a finite-to-one function such that $f(\mathscr{F})$ is Fréchet iff $\mathscr{F}$ is meagre, that is, the set of the characteristic functions of the members of $\mathscr{F}$ is a meagre subset of the space $2^{\omega}$.

The principle of near coherence of filters, $\mathrm{NCF}$, says that any two filters (equivalently: ultrafilters) are nearly coherent. Blass and Laflamme 5] showed that $\mathfrak{u}<\mathfrak{g}$ implies FD, and that FD implies NCF. The purpose of this paper is to show that NCF does not imply FD.

Main Theorem. "NCF and not FD" is consistent relative to ZFC.

Since NCF is equivalent to " $\beta \mathbb{R}_{+} \backslash \mathbb{R}_{+}$has only one composant" and to "the ideal of compact operators on a Hilbert space is not the sum of two proper subideals" [3] and since FD is weaker than "there are just four slenderness classes of abelian groups" (by [5]; actually by [14 they are equivalent), our result says that it is possible that $\beta \mathbb{R}_{+} \backslash \mathbb{R}_{+}$has only one composant and the ideal of compact operators

Received by the editors November 16, 2006.

2000 Mathematics Subject Classification. Primary 03E35, 03E17, 03E75, 54D40.

The first author was partially supported by the Landau Center.

The second author's research was partially supported by the United States-Israel Binational Science Foundation (Grant no. 2002323). This is the second author's publication no. 894. 
on a Hilbert space is not the sum of two proper subideals, and yet there are more than four slenderness classes of abelian groups.

We also looked at the splitting number $\mathfrak{s}$ (see [1, 1.3.5]) in our models of $\mathrm{NCF}$ and not FD, because by [14, FD together with $\mathfrak{s}>\mathfrak{u}$ implies $\mathfrak{u}<\mathfrak{g}$, and we wanted to know whether $\mathfrak{s}$ also has an influence in our non-implication. We give two types of models of NCF and not FD, one with $\mathfrak{s}=\aleph_{2}$ and one where we do not know the splitting number. The hope is that the elbow room in our construction will at some time help to solve the open problem of whether $\mathfrak{u}<\mathfrak{g}$ is strictly stronger than FD.

Before we give an outline of the forcing construction, let us first review the known models of FD. $P$-points and two cardinal characteristics play an important rôle in our topic: We write $A \subseteq^{*} B$ iff $A \backslash B$ is finite. An ultrafilter $\mathscr{U}$ is called a $P$-point if for every $\gamma<\aleph_{1}$, for every $A_{i} \in \mathscr{U}, i<\gamma$, there is some $A \in \mathscr{U}$ such that for all $i<\gamma, A \subseteq^{*} A_{i}$; such an $A$ is called a pseudo-intersection or a diagonalisation of the $A_{i}, i<\gamma$. A notion of forcing $\mathbb{P}$ preserves an ultrafilter $\mathscr{U}$ iff $\vdash_{\mathbb{P}}$ " $\left(\forall X \in[\omega]^{\aleph_{0}}\right)(\exists Y \in \mathscr{U})(Y \subseteq X \vee Y \subseteq \omega \backslash X)$ ", and in the contrary case we say "P्P destroys $\mathscr{U}$ ". If $\mathbb{P}$ preserves $\mathscr{U}$ and $\mathscr{U}$ is a $P$-point, then $\mathscr{U}$ stays a $P$-point [6. Lemma 3.2].

$\mathscr{B} \subseteq \mathscr{U}$ is a base for $\mathscr{U}$ if for every $X \in \mathscr{U}$ there is some $Y \in \mathscr{B}$ such that $Y \subseteq X$. The cardinal $\mathfrak{u}$ is the smallest cardinal of a base for a non-principal ultrafilter.

A subset $\mathscr{G}$ of $[\omega]^{\omega}$ is called groupwise dense if $(\forall X \in \mathscr{G})\left(\forall Y \subseteq^{*} X\right)(Y \in \mathscr{G})$, and for every partition of $\omega$ into finite intervals $\left\{\left[\pi_{i}, \pi_{i+1}\right): i \in \omega\right\}$ there is an infinite set $A$ such that $\bigcup\left\{\left[\pi_{i}, \pi_{i+1}\right): i \in A\right\} \in \mathscr{G}$. The groupwise density number, $\mathfrak{g}$, is the smallest number of groupwise dense families with empty intersection.

The only models of NCF that have been known so far are also models of FD and $\mathfrak{u}<\mathfrak{g}$, which is possibly strictly stronger than FD. A ground model with $\mathrm{CH}$ is extended by an iterated forcing $\left\langle\mathbb{P}_{\beta}, \mathbb{Q}_{\alpha}: \beta \leq \gamma, \alpha<\gamma\right\rangle$ that is built in the usual way: The iterand $\mathbb{Q}_{\alpha}$ is a $\mathbb{P}_{\alpha}$-name and $\mathbb{P}_{\alpha+1}=\mathbb{P}_{\alpha} * \mathbb{Q}_{\alpha}$, and at limits we build $\mathbb{P}_{\alpha}$ with countable supports. The iterands are proper forcings that preserve at least one, indeed all $P$-points, and thus keep $\mathfrak{u}$ small. (If $\mathfrak{u}<\mathfrak{d}$, which follows from NCF, then by Ketonen [11 every filter witnessing $\mathfrak{u}$ is a $P$-point, so we do not have to worry whether there is some non- $P$ ultrafilter with a base of a smaller size. Also, since $\mathfrak{u}=\aleph_{1}$ is the minimum possible, these worries are unnecessary.) Let us write $\mathbf{V}_{\alpha}$ for $\mathbf{V}^{\mathbb{P}_{\alpha}}$, an arbitrary extension by a $\mathbb{P}_{\alpha}$-generic filter $G_{\alpha}$. Although $\mathfrak{u}$ is kept small, at least at stationarily many limit steps $\alpha<\aleph_{2}$ of cofinality $\aleph_{1}$ the next iterand adds a real that has supersets in all groupwise dense sets in $\mathbf{V}_{\alpha}$ and thus $\mathfrak{g}=\aleph_{2}[5]$.

Some types of such models of $\mathfrak{u}<\mathfrak{g}$ are known: An iteration of length $\aleph_{2}$ with countable support of Blass-Shelah forcing over a ground model of $\mathrm{CH}$ [6] gives $\aleph_{1}=\mathfrak{u}<\mathfrak{s}=\mathfrak{g}=2^{\aleph_{0}}=\aleph_{2}$, and an iteration of length $\aleph_{2}$ with countable support of Miller forcing over a ground model of CH [7] gives $\aleph_{1}=\mathfrak{u}=\mathfrak{s}<\mathfrak{g}=2^{\aleph_{0}}=\aleph_{2}$. A third type of model of $\mathfrak{u}<\mathfrak{g}$ is given by a countable support iteration of Matet forcing 3 . Other proper tree forcings that preserve $P$-points can be interwoven into the iteration and, as long as at stationarily many steps of cofinality $\aleph_{1}$ a real is added that has a superset in each groupwise dense family in the intermediate model, the outcome is $\mathfrak{u}<\mathfrak{g}$.

Now our proof of the main theorem modifies these constructions. First: We preserve only one arbitrary $P$-point $\mathscr{E} \in \mathbf{V}_{0}$ that will be fixed forever, and we destroy many others. 
We try to specify what we mean when we use one of the ambiguous expressions "stage $\alpha$ " or "step $\alpha$ "; When we say "at stage $\alpha$ we have" or something similar it means "in $\mathbf{V}^{\mathbb{P}_{\alpha}}$ holds". When we say "at stage $\alpha$ we do" it refers to the choice of $\mathbb{Q}_{\alpha}$, which is a part of $\mathbb{P}_{\alpha+1}=\mathbb{P}_{\alpha} * \mathbb{Q}_{\alpha}$. Second: We let $S_{1}^{2}=\left\{\alpha \in \aleph_{2}: \operatorname{cf}(\alpha)=\aleph_{1}\right\}$. We build up one non-meagre non-nearly ultra filter $\mathscr{A}$ generated by $\left\{A_{\alpha}: \alpha \notin S_{1}^{2}\right\}$. In a stage $\alpha \in \aleph_{2} \backslash S_{1}^{2}$ we let $\mathbb{Q}_{\alpha}$ diagonalise $\mathscr{A}$ 's initial segment $\mathscr{A}_{\alpha}:=$ the filter generated in $\mathbf{V}_{\alpha}$ by $\left\{A_{\beta}: \beta<\alpha, \beta \notin S_{1}^{2}\right\}$ and let $A_{\alpha}$ be a subset of the complement of a diagonalisation built from certain blocks. Looking at sufficiently many combinations of infinitely many blocks guarantees $\mathscr{A}=\mathscr{A}_{\omega_{2}}$ will not be meagre in the end. Also $\mathscr{A}$ will be very far from being ultra, because at any time it contains a tree of $2^{\aleph_{1}}$ mutually non-nearly coherent core filters $\Phi(\mathscr{U})$ (see Definition (3.4) among its supersets, and at stages $\alpha \in \aleph_{2} \backslash S_{1}^{2}$ the filter $\mathscr{A}_{\alpha}$ even has a pseudo-intersection (see Definition 4.1) in $\mathbf{V}_{\alpha}$ (if $\operatorname{cf}(\alpha)=\omega$ and $\alpha$ is the limit of a sequence in $S_{1}^{2}$, then we take a cofinal sequence $\beta_{n}$ converging to $\alpha$ and take a pseudo-intersection of the centred set $\left\{X_{\beta_{n}}: n \in \omega\right\}$; note that by the definition of iterated forcing the sequence $\left\langle X_{\beta_{n}}: n \in \omega\right\rangle \in \mathbf{V}^{\mathbb{P}_{\alpha}}$; in the successor case $\alpha=\beta+1$, $\beta \notin S_{1}^{2}$ we use the induction hypothesis and the fact that $\left\{A_{\beta}\right\} \cup \mathscr{A}_{\beta}$ is centred; if $\alpha=\beta+1, \beta \in S_{1}^{2}$, then $X_{\alpha}$ is a pseudo-intersection of $\mathscr{A}_{\beta}$; the case of $\operatorname{cf}(\alpha)=\omega$ and $\alpha$ has a maximal predecessor in $S_{1}^{2}$ is similar) and at stages $\alpha \in S_{1}^{2}$ the filter $\mathscr{A}_{\alpha}$ has a pseudo-intersection in $\mathbf{V}_{\alpha+1}$. We strengthen the latter properties of $\mathscr{A}_{\alpha}$ to a property of every two stages $\beta<\alpha, \beta, \alpha \in \aleph_{2} \backslash S_{1}^{2}$ that is preserved in the iteration and that will allow us to work with stable ordered-union ultrafilters $\mathscr{U}$ on the set $\mathbb{F}$ of all finite, non-empty subsets of $\omega$. The strengthening will be the technical property (P5) of the iteration in Section 5. It prevents the misfortune that for some $\alpha \in S_{1}^{2}, \mathscr{A}_{\alpha}$ is all of a sudden nearly ultra.

Third: We get NCF with the aid of a diamond and special iterands. A diamond sequence on $S_{1}^{2}$ is a sequence $\left\langle S_{\alpha}: \alpha \in S_{1}^{2}\right\rangle$ such that for all $X \subseteq \aleph_{2}$ the set $\left\{\alpha \in S_{1}^{2}: X \cap \alpha=S_{\alpha}\right\}$ is stationary. $\diamond\left(S_{1}^{2}\right)$ says that there is a diamond sequence on $S_{1}^{2}$.

The tricky part is to find suitable iterands $\mathbb{Q}_{\alpha}$ for $\alpha \in S_{1}^{2}: \mathbb{Q}_{\alpha}$ shall preserve $\mathscr{E}$, shall make the ultrafilter handed down by the diamond to be nearly coherent to $\mathscr{E}$ and shall diagonalise $\mathscr{A}_{\alpha}$ by adding a pseudo-intersection $X_{\alpha} \in \mathbf{V}_{\alpha+1}$. Thus in the whole extension $\mathscr{A}$ is not mapped by any finite-to-one function to an ultrafilter.

We divide the proof of the Main Theorem into four sections: First we deal with the iterands for the stages $\alpha \notin S_{1}^{2}$. In Section 3 we work on the iterands for the stages $\alpha \in S_{1}^{2}$. In Section 4 we introduce pseudo-intersections and witnesses. In Section 5 we put the iteration together. In Section 6 we consider the values of the entries of Cichon's diagramme and of $\mathfrak{s}, \mathfrak{r}, \mathfrak{u}, \mathfrak{g}, \mathfrak{h}$ and other cardinal invariants in the two types of models of "NCF and not FD" from Section 5.

\section{The iterands $\mathbb{Q}_{\alpha}$ FOR $\alpha \notin S_{1}^{2}$}

In the stages $\alpha \notin S_{1}^{2}$ of the iteration $\mathbb{Q}_{\alpha}$ shall add some set $A_{\alpha}$ to $\mathscr{A}_{\alpha}$, the filter generated by $\left\{A_{\beta}: \beta<\alpha\right\}$, so that these additions will guarantee that in the end $\mathscr{A}=\mathscr{A}_{\mathbb{N}_{2}}$ is not meagre. Any forcing that diagonalises all groupwise dense sets in the ground model would accomplish this task. However, we consider here only two candidates: One is Blass-Shelah forcing $\mathbb{Q}$ (see [6] or [1, pages $370 \mathrm{ff}$.$] ) and the$ second is Matet forcing. 
Why just these two? Using the first in unboundedly many steps makes $\mathfrak{s}=\aleph_{2}$ in the final model, whereas taking coboundedly often the latter possibly keeps $\mathfrak{s}$ small.

Now we review Matet forcing, since we shall also use a suborder of it later in the choice of the iterands $\mathbb{Q}_{\alpha}$ for $\alpha \in S_{1}^{2}$.

We let $\mathbb{F}$ be the collection of all non-empty finite subsets of $\omega$. For $a, b \in \mathbb{F}$ we write $a<b$ if $(\forall n \in a)(\forall m \in b)(n<m)$. We will work with proper filters on $\mathbb{F}$, i.e. subsets of $\mathscr{P}(\mathbb{F})$ that are closed under binary intersections and supersets and do not contain the empty set. A sequence $\bar{c}$ of members of $\mathbb{F}$ is called unmeshed if for all $n, c_{n}<c_{n+1}$. The set $(\mathbb{F})^{\omega}$ denotes the collection of all infinite unmeshed sequences in $\mathbb{F}$. If $\bar{c}$ is a sequence in $(\mathbb{F})^{\omega}$, we write $(\mathrm{FU})^{\omega}(\bar{c})$ for the set of all unmeshed sequences whose members are finite unions of some of the $c_{n}$ 's and we write $\mathrm{FU}(\bar{c})$ for the set of all finite unions of members of $\bar{c}$. The symbol $={ }^{*}$ denotes equality up to finitely many exceptions.

Definition 2.1. Given $\bar{c}$ and $\bar{d}$ in $(\mathbb{F})^{\omega}$, we say that $\bar{d}$ is a condensation of $\bar{c}$ and we write $\bar{d} \sqsubseteq \bar{c}$ if $\bar{d} \in(\mathrm{FU})^{\omega}(\bar{c})$. We say $\bar{d}$ is almost a condensation of $\bar{c}$ and we write $\bar{d} \Xi^{*} \bar{c}$ iff there is an $n$ such that $\left\langle d_{t}: t \geq n\right\rangle$ is a condensation of $\bar{c}$. If there is some $A \in[\omega]^{\aleph_{0}}$ such that $X={ }^{*} \bigcup\left\{c_{n}: n \in A\right\}$, then we let $\bar{c} \cap X$ be defined as $\left\langle c_{n} \cap X: n \in \omega, c_{n} \cap X \neq \emptyset\right\rangle$.

Note that $\bar{c} \cap X$, when defined, is almost a condensation of $\bar{c}$.

Definition 2.2. 1. In the Matet forcing, $\mathbb{M}$, the conditions are pairs $(w, \bar{c})$ such that $w \in \mathbb{F}$ and $\bar{c} \in(\mathbb{F})^{\omega}$ and $w<c_{0}$. The forcing order is $\left(w^{\prime}, \bar{c}^{\prime}\right) \geq(w, \bar{c})$ iff $w \subseteq w^{\prime}$ and $w^{\prime} \backslash w$ is a union of finitely many of the $c_{n}$ and $\bar{c}^{\prime}$ is a condensation of $\bar{c}$.

2. The set $\mathbb{M}^{\mathrm{pr}}$ of pure conditions of $\mathbb{M}$ is the set of conditions with $w=\emptyset$. In this case we write $\bar{c}$ instead of $(\emptyset, \bar{c})$. For two pure conditions we let $\bar{c} \leq^{*} \bar{d}$ iff $\bar{c} \sqsupseteq^{*} \bar{d}$. We let $\operatorname{set}(\bar{c})=\bigcup\left\{c_{n}: n \in \omega\right\}$.

In $[3$ it is shown that $\mathbb{M}$ is proper. In unpublished work, Blass and Laflamme independently have shown that $\mathbb{M}$ preserves $P$-points. Eisworth's work ( 9 , Theorem 4] or Theorem 3.5 below) implies this result, as we shall explain below.

We explain why we prefer the Matet forcing: Given a stable ordered union ultrafilter (see Definition 3.1) $\mathscr{U}$ on $\mathbb{F}$, we can thin out the Matet partial order to a $\sigma$-centred subforcing. In the thinning process Hindman's theorem $([10,3.3]$, below Theorem 3.2) is used, and we do not know how to apply it unless we have forgetful [17, 1.2.5] forcings. "Forgetful" [17, Definition 1.2.5 (3)] means that the possible strengthenings of the part $c_{i} \subseteq[f(i), f(i+1))$ of a condition $(w, \bar{c})$ do not depend on the part of the condition outside $[f(i), f(i+1))$; in particular not on the part of the condition below $f(i)$, as in the tree forcings, like Laver, Sacks, Miller or the more general examples from Chapter 1.3 of [17. Moreover in some technical parts of our proof, again for the mentioned property (P5), we shall use the following property: For every condition $\left(w^{\prime}, \bar{c}^{\prime}\right)$ in the Matet forcing, the generic real $X=\bigcup\{w:(\exists \bar{c})((w, \bar{c}) \in G)\}$ coincides up to finitely many exceptions with the union over a suitable infinite set of blocks $c_{n}^{\prime}$ or there is some $(w, \bar{c}) \in G$ such that $\bar{c}$ and $\bar{c}^{\prime}$ do not have a common almost condensation. In the next section we consider subforcings in which the second part of this disjunction is excluded. 


\section{3. $\sigma$-CENTRED SUBFORCINGS OF $\mathbb{M}$}

In this section we look for the iterands $\mathbb{Q}_{\alpha}$ for $\alpha \in S_{1}^{2}$. To define these (necessarily non-complete, as they will destroy some $P$-points) subforcings of $\mathbb{M}$, we first introduce some properties of filters on the set $\mathbb{F}$ of all non-empty finite subsets for $\omega$. Our nomenclature follows Blass [2] and Eisworth [9].

Note that if we used the Blass-Shelah forcing or the Matet forcing as iterand $\mathbb{Q}_{\alpha}$ at stationarily many stages $\alpha \in S_{1}^{2}$, then we would get $\mathfrak{u}<\mathfrak{g}$, which implies FD.

Definition 3.1. A non-principal filter $\mathscr{F}$ on $\mathbb{F}$ is said to be an ordered-union filter if it has a basis of sets of the form $\mathrm{FU}(\bar{d})$ for $\bar{d} \in(\mathbb{F})^{\omega}$. Let $\mu$ be an uncountable cardinal. An ordered-union filter is said to be $<\mu$-stable if, whenever it contains $\mathrm{FU}\left(\bar{d}_{\alpha}\right)$ for $\bar{d}_{\alpha} \in(\mathbb{F})^{\omega}, \alpha<\kappa$, for some $\kappa<\mu$, then it also contains some $\mathrm{FU}(\bar{e})$ for some $\bar{e}$ that is almost a condensation of $\bar{d}_{\alpha}$ for $\alpha<\kappa$. For " $<\aleph_{1}$-stable" we say "stable".

Ordered-union ultrafilters need not exist, as their existence implies the existence of $Q$-points [2] and there are models without $Q$-points [16]. With the help of Hindman's theorem one shows that under $\operatorname{MA}\left(\sigma\right.$-centred) stable (even $<2^{\omega}$-stable) ordered-union ultrafilters exist 2]. We will construct suitable stable ordered-union ultrafilters for the choice of $\mathbb{Q}_{\alpha}, \alpha \in S_{1}^{2}$, by induction on $\aleph_{1}$ using $\mathrm{CH}$ and Hindman's theorem:

Theorem 3.2 (Hindman, [10, Corollary 3.3]). If the set $\mathbb{F}$ is partitioned into finitely many pieces, then there is a set $\bar{d} \in(\mathbb{F})^{\omega}$ such that $\mathrm{FU}(\bar{d})$ is included in one piece.

The theorem also holds if instead of $\mathbb{F}$ we partition only $\mathrm{FU}(\bar{c})$ for some $\bar{c} \in(\mathbb{F})^{\omega}$; the homogeneous sequence $\bar{d}$ given by the theorem is then a condensation of $\bar{c}$.

Definition 3.3. Given an ordered-union ultrafilter $\mathscr{U}$ on $\mathbb{F}$ we let $\mathbb{M}(\mathscr{U})$ consist of all pairs $(s, \bar{c}) \in \mathbb{M}$, such that $s \in \mathbb{F}$ and $\mathrm{FU}(\bar{c}) \in \mathscr{U}$. The forcing order is the same as in the Matet forcing.

It is well known 13, 3 that Matet forcing $\mathbb{M}$ can be decomposed into two steps $\mathbb{P} * \mathbb{M}(\underset{\sim}{\mathscr{U}})$, such that $\mathbb{P}$ is $\aleph_{1}$-closed (that is, every descending sequence of conditions of countable length has a lower bound) and adds a stable ordered-union ultrafilter $\mathscr{U}$ on the set $\mathbb{F}$.

In order to state a preservation property of $\mathbb{M}(\mathscr{U})$, we need the following definition.

Definition 3.4. Let $\mathscr{U}$ be a filter on $\mathbb{F}$. The core of $\mathscr{U}$ is the filter $\Phi(\mathscr{U})$ such that

$$
X \in \Phi(\mathscr{U}) \text { iff }(\exists \mathrm{FU}(\bar{c}) \in \mathscr{U})\left(\bigcup_{n \in \omega} c_{n} \subseteq X\right) .
$$

If $\mathscr{U}$ is ultra on $\mathbb{F}$, then $\Phi(\mathscr{U})$ is not diagonalised (see [9, Prop. 2.3]) and also all finite-to-one images of $\Phi(\mathscr{U})$ are not diagonalised (the same proof). So $\Phi(\mathscr{U})$ is not meagre.

The Rudin-Blass ordering on filters on $\omega$ is defined as follows: Let $\mathscr{F} \leq_{R B} \mathscr{G}$ iff there is a finite-to-one $f$ such that $f(\mathscr{F}) \subseteq f(\mathscr{G})$. The following property of stable ordered-union ultrafilters $\mathscr{U}$ will be important for our proof:

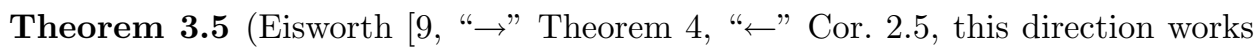
also with non- $P$ ultrafilters] $)$. Let $\mathscr{U}$ be a stable ordered-union ultrafilter on $\mathbb{F}$ and 
let $\mathscr{V}$ be a P-point. Iff $\mathscr{V} \Varangle_{R B} \Phi(\mathscr{U})$, then $\mathscr{V}$ continues to generate an ultrafilter after we force with $\mathbb{M}(\mathscr{U})$.

In the decomposition $\mathbb{M}=\mathbb{P} * \mathbb{M}(\mathscr{U})$, the stable ordered-union ultrafilter $\mathscr{U}$ in the intermediate model fulfills $\Phi(\mathscr{U}) \mathbb{Z}_{\mathrm{RB}} \mathscr{V}$ for any $P$-point $\mathscr{V}$ in the ground model, and hence by Theorem 3.5, $\mathbb{M}$ preserves $P$-points.

We shall not add $\mathscr{U}$ by forcing, but working with a $\complement^{*}$-descending sequence $\bar{c}^{\alpha}$, $\alpha<\aleph_{1}$, with the property that $\mathrm{FU}\left(\bar{c}^{\alpha}\right), \alpha<\aleph_{1}$, generates an ultrafilter $\mathscr{U}$ on $\mathbb{F}$. Then this is a stable ordered-union ultrafilter.

\section{Filters With PSEUdo-intersections}

In this section we work with some properties of filters on $\omega$, in the direction of building a non-meagre non-nearly-ultra filter $\mathscr{A}$.

Definition 4.1. Let $\mathscr{A} \subseteq[\omega]^{\aleph_{0}}$ be such that for all $n$ for all $X_{0}, \ldots, X_{n} \in \mathscr{A}$, $\bigcap_{i \leq n} X_{i}$ is infinite. This is called " $\mathscr{A}$ is centred" or " $\mathscr{A}$ has the finite intersection property".

(a) By fil $(\mathscr{A})$ we denote the filter on $\omega$ generated by $\mathscr{A} \cup\{\omega \backslash n: n<\omega\}$. Later, when we are working in the context of an intermediate forcing extension $\mathbf{V}_{\alpha}$, the non-absolute definition $\operatorname{fil}(\mathscr{A})$ is to be interpreted in that $\mathbf{V}_{\alpha}$.

(b) $X \in[\omega]^{\aleph_{0}}$ is a pseudo-intersection of $\mathscr{A}$ (or diagonalises $\mathscr{A}$ ) if for all $A \in \mathscr{A}$, $X \subseteq^{*} A$.

Note that $X \in[\omega]^{\aleph_{0}}$ is a pseudo-intersection of $\mathscr{A}$ if it is a pseudo-intersection of fil $(\mathscr{A})$. Only centred sets can have pseudo-intersections.

\section{Definition 4.2.}

(1) $\mathscr{R}^{*}=\left\{R \subseteq \omega \times \omega:(\forall m)\left(\exists^{<\aleph_{0}} n\right)(m R n) \wedge(\forall n)\left(\exists<\aleph_{0} m\right)(m R n)\right\}$. The quantifier $\exists<\aleph_{0}$ means that there are finitely many and at least one.

(2) For $R, S \in \mathscr{R}^{*}$ we let $R^{-1}=\{(n, m):(m, n) \in R\}$ and we let $R \circ S=$ $\{(m, r):(\exists n)((m, n) \in R \wedge(n, r) \in S)\}$. Note that the order is different from the one known in the composition of functions: We first "map" with $R$, then with $S$. For $\mathscr{R} \subseteq \mathscr{R}^{*}$ and $S \in \mathscr{R}^{*}$ we let $S \circ \mathscr{R}=\{S \circ R: R \in \mathscr{R}\}$.

(3) For $A \subseteq \omega, R \in \mathscr{R}^{*}$ we let $R(A)=\{n: m R n, m \in A\}$.

(4) For $\bar{c}=\left\langle c_{n}: n \in \omega\right\rangle \in(\mathbb{F})^{\omega}, R \in \mathscr{R}^{*}$ we let $R(\bar{c})=\left\langle R\left(c_{n}\right): n \in \omega\right\rangle$. This can be meshed or even be not pairwise disjoint (that is, not unmeshed), but it does not matter.

Note that $R^{-1}$ and $R \circ S$ are also in $\mathscr{R}^{*}$. The next definition will play a crucial rôle in the iteration.

Definition 4.3. We say $(\bar{c}, \mathscr{R})$ is a witness over $\mathscr{A}$ when:

(a) $\mathscr{A} \subseteq[\omega]^{\aleph_{0}}$ is centred,

(b) $\bar{c} \in(\mathbb{F})^{\omega}$,

(c) $\mathscr{R}$ is a countable subset of $\mathscr{R}^{*}$,

(d) $\mathscr{R} \neq \emptyset$,

(e) for every $R \in \mathscr{R}$, the set $R(\operatorname{set}(\bar{c}))$ is a pseudo-intersection of $\mathscr{A}$.

The purpose of $R \in \mathscr{R}^{*}$ is to increase infinite sets in a gentle manner, as with finite-to-one functions: $f(\mathscr{F})=\left\{X: f^{-1} X \in \mathscr{F}\right\}=\{X: R(X) \in \mathscr{F}\}$, where $x R y$ iff $f(y)=x$. Since $f$ is a finite-to-one function, we have $R \in \mathscr{R}$. Iff for every 
$R \in \mathscr{R}^{*}$ there is some $X \in \mathscr{F}$ such that $R(X) \notin \mathscr{V}$, then $\mathscr{F}$ is not Rudin-Blass below $\mathscr{V}$. We shall use the "if"-direction of this criterion for $\mathscr{F}=\Phi(\mathscr{U})$ and $\mathscr{V}=\mathscr{E}$ in the final section.

However, our main use of $\mathscr{R}^{*}$ is the following: We use countable subsets $\mathscr{R}$ of $\mathscr{R}^{*}$ to map pseudo-intersections of $\mathscr{A}_{\alpha}$ to other pseudo-intersections of $\mathscr{A}_{\alpha}$, as in the definition of "witness". The union of all used countable parts (that is, $\bigcup_{\varepsilon<\aleph_{1}} \mathscr{R}_{\varepsilon}$ from Lemma 5.4 note we require $\zeta<\varepsilon \rightarrow \mathscr{R}_{\zeta} \subseteq \mathscr{R}_{\varepsilon}$ ) at a limit step $\alpha \in S_{1}^{2}$ does not exhaust $\left(\mathscr{R}^{*}\right)^{\mathbf{V}_{\alpha}}$, since $\mathscr{A}_{\alpha}$ is centred and there are $\{(n, 2 n): n \in \omega\}$ and $\{(n, 2 n+1): n \in \omega\}$, which can never be both elements of $\mathscr{R}$, such that there is a $\bar{c} \in(\mathbb{F})^{\omega}$ with $(\bar{c}, \mathscr{R})$ witnessing over $\mathscr{A}_{\beta}$ for some $\beta<\alpha$. However, it does so modulo composition with "shifts" $R_{\bar{c}, \bar{d}}=\left\{(m, n):(\exists i)\left(m \in c_{i} \wedge n \in d_{i}\right)\right\}$ for $\bar{c}, \bar{d} \in(\mathbb{F})^{\omega}$. This will follow from the technical requirement $(\gamma)$ in the proof of Lemma 5.4

Lemma 4.4. (1) Assume that $\mathbf{V}$ is a transitive class in $\mathbf{V}^{+}$. If in $\mathbf{V},(\bar{c}, \mathscr{R})$ is a witness over $\mathscr{A}$, then this also holds in $\mathbf{V}^{+}$.

(2) $(\bar{c}, \mathscr{R})$ witnesses over $\mathscr{A}$ iff for every $A \in \mathscr{A},(\bar{c}, \mathscr{R})$ witnesses over $\{A\}$.

(3) $(\bar{c}, \mathscr{R})$ witnesses over $\mathscr{A}$ iff $(\bar{c}, \mathscr{R})$ witnesses over $\operatorname{fil}(\mathscr{A})$.

(4) If $(\bar{c}, \mathscr{R})$ witnesses over $\mathscr{A}$ and $\bar{d} \sqsubseteq^{*} \bar{c}$, then $(\bar{d}, \mathscr{R})$ witnesses over $\mathscr{A}$.

(5) If $\mathscr{R} \subseteq \mathscr{R}^{*}$ and $\bar{c}, \bar{d} \in(\mathbb{F})^{\omega}$ and $R=R_{\bar{c}, \bar{d}}=\left\{(m, n):(\exists i)\left(m \in c_{i} \wedge n \in d_{i}\right)\right\}$, then $R(\bar{c})=\bar{d}$ and $R^{-1}=R_{\bar{d}, \bar{c}}$ and $\left(\bar{d}, R_{\bar{d}, \bar{c}} \circ \mathscr{R}\right)$ witnesses over $\mathscr{A}$ iff $(\bar{c}, \mathscr{R})$ witnesses over $\mathscr{A}$.

Proof. (1) The definition of witnessing contains only bounded quantifiers and an existential quantifier. Items (2), (3), and (4) are obvious. Proof of (5): We compute $R^{-1}\left(d_{k}\right)=\left\{(n, m): \exists i\left(m \in d_{i} \wedge n \in c_{i}\right)\right\}\left(d_{k}\right)=c_{k}$ so $R^{-1}=R_{\bar{d}, \bar{c}}$. Although $R \circ R^{-1}=\left\{(n, m):(\exists i)\left(n, m \in c_{i}\right)\right\}$ is in general not a subset of the diagonal, we have $\left(R \circ R^{-1}\right)\left(c_{n}\right)=c_{n}$. Now $\left(R^{-1} \circ S\right)\left(d_{k}\right)=\left\{(m, r): \exists n\left((m, n) \in R^{-1} \wedge(n, r) \in\right.\right.$ $S)\}\left(d_{k}\right)=S\left(R^{-1}\left(d_{k}\right)\right)=S\left(c_{k}\right)$. So we have $(\forall A \in \mathscr{A})(\forall S \in \mathscr{R})\left(S(\operatorname{set}(\bar{c})) \subseteq^{*} A\right)$ iff $(\forall A \in \mathscr{A})(\forall S \in \mathscr{R})\left(R^{-1} \circ S(\operatorname{set}(\bar{d})) \subseteq \subseteq^{*} A\right)$.

\section{The ITERATiON}

We start with a ground model $\mathbf{V}$ that fulfills $\mathrm{CH}$ and $\diamond\left(S_{1}^{2}\right)$ (and hence $2^{\aleph_{1}}=\aleph_{2}$ ).

In a countable support iteration of proper forcings of iterands of size $\leq \aleph_{1}$ each real appears in a $\mathbf{V}_{\alpha}$ for some $\alpha$ with countable cofinality, and a reflection property ensures that each ultrafilter $\mathscr{U}$ in the final model has $\aleph_{1}$-club many $\alpha \in \aleph_{2}$ such that $\mathscr{U} \cap V_{\alpha}$ has a $\mathbb{P}_{\alpha}$-name and is an ultrafilter in $\mathbf{V}_{\alpha}$ (see [6, Item 5.6 and Lemma 5.10]). A subset of $\aleph_{2}$ is called $\aleph_{1}$-club if it is unbounded in $\aleph_{2}$ and closed under suprema of strictly ascending sequences of lengths $\aleph_{1}$. By well-known techniques based on coding $\mathbb{P}_{\alpha}$-names for ultrafilters as subsets of $\aleph_{2}$ (e.g., such a coding is carried out in 15. Claim 2.8]) and based on the maximal principle (see, e.g., 12, Theorem

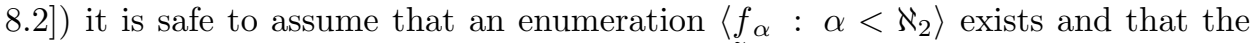
$\diamond\left(S_{1}^{2}\right)$-sequence $\left\langle\underset{\sim}{S_{\alpha}}: \alpha \in S_{1}^{2}\right\rangle$ gives an $\aleph_{1}$-club set of $\mathbb{P}_{\alpha}$-names $\underset{\sim}{S_{\alpha}}$ for an ultrafilter in $\mathbf{V}^{\mathbb{P}_{\alpha}}$ such that for any ultrafilter $\mathscr{U} \in V^{\mathbb{P}_{\aleph_{2}}}$ there are stationarily many $\alpha \in S_{1}^{2}$ with $\mathscr{U} \cap V^{\mathbb{P}_{\alpha}}=S_{\alpha}$. For names $\underset{\sim}{x}$ and objects $x$ we use the rule $\underset{\sim}{x}[G]=x$.

We fix a diamond sequence $\left\langle\underset{\sim}{S_{\alpha}}: \alpha \in S_{1}^{2}\right\rangle$. Only $S_{\alpha}$ gets a second letter: $S_{\alpha}=\mathscr{D}$ to make clearer that it is an ultrafilter. We also fix a $P$-point $\mathscr{E} \in \mathbf{V}$ that will be preserved throughout our iteration. Let $f_{\alpha}, \alpha \in \aleph_{2} \backslash S_{1}^{2}$, be an enumeration of all $\mathbb{P}_{\aleph_{2}}$-names for finite-to-one, monotone, surjective functions from $\omega$ to $\omega$, each 
appearing cofinally often, such that $f_{\alpha}$ is a $\mathbb{P}_{\alpha}$-name. We assume that $\pi_{\alpha}$ is a $\mathbb{P}_{\alpha}$-name such that for all evaluations $f_{\alpha}, \pi_{\alpha}$ of $\underset{\sim}{f_{\alpha}},{\underset{\sim}{\alpha}}_{\alpha}$ respectively, $\pi_{\alpha}(0)=0$, $\pi_{\alpha}(n+1)=\max \left(f_{\alpha}^{-1}(n)\right)+1$.

We construct (carefully) by induction on $\alpha \leq \aleph_{2}$ a countable support iteration of proper forcings $\left\langle\mathbb{P}_{\alpha}, \mathbb{Q}_{\beta}: \beta<\aleph_{2}, \alpha \leq \aleph_{2}\right\rangle$ and two sequences of names $\left\langle{ }_{\sim} A_{\alpha}\right.$ : $\left.\alpha \in \aleph_{2} \backslash S_{1}^{2}\right\rangle$ and $\left\langle\underset{\sim}{X}: \alpha \in S_{1}^{2}\right\rangle$ such that

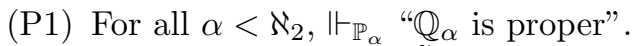

(P2) For all $\alpha \leq \aleph_{2}, \Vdash_{\mathbb{P}_{\alpha}}$ "fil $(\mathscr{E})$ is ultra".

(P3) For $\alpha \in \aleph_{2} \backslash S_{1}^{2}, A_{\alpha}$ is a $\mathbb{P}_{\alpha+1}$ name. We write $A_{\alpha}=\underset{\sim}{A_{\alpha}}\left[G_{\alpha+1}\right]$. For each $\alpha \in \aleph_{2} \backslash S_{1}^{2},\left\{A_{\beta}: \beta \in \alpha \backslash S_{1}^{2}\right\}$ is centred and $f_{\alpha}\left(A_{\alpha}\right) \neq^{*} \omega$. We let $\mathscr{A}_{\alpha}=\operatorname{fil}\left(\left\{A_{\beta}: \beta \in \alpha \backslash S_{1}^{2}\right\}\right)$. So $A_{\alpha}$ shows that $f_{\alpha}\left(\mathscr{A}_{\alpha+1}\right)$ is not the Fréchet filter.

(P4) For $\alpha \in S_{1}^{2},{\underset{\sim}{\alpha}}_{\alpha}$ is a $\mathbb{P}_{\alpha+1}$-name. Let $\mathscr{A}_{\alpha}$ be a $\mathbb{P}_{\alpha}$-name for $\mathscr{A}_{\alpha}$. If $\alpha \in S_{1}^{2}$ and if $S_{\alpha}$ is a $\mathbb{P}_{\alpha}$-name $\mathscr{D}$ for an ultrafilter in $V^{\mathbb{P}_{\alpha}}$, then $\vdash_{\mathbb{P}_{\alpha+1}}$ " $\mathscr{D}$ and $\operatorname{fil}(\mathscr{E})$ are nearly coherent, and $X_{\alpha}$ is a pseudo-intersection of $\mathscr{A}_{\alpha}$ ".

(P5) For $\beta<\gamma, \beta, \gamma \in \aleph_{2} \backslash S_{1}^{2}$, if $G_{\gamma} \subseteq \mathbb{P}_{\gamma}$ is generic over $\mathbf{V}$ and $G_{\beta}=\mathbb{P}_{\beta} \cap G_{\gamma}$, then

$$
\begin{aligned}
& \text { if } \mathbf{V}_{\beta} \models "(\bar{c}, \mathscr{R}) \text { is a witness over } \mathscr{A}_{\beta} ", \\
& \text { then } \mathbf{V}_{\gamma} \models "(\exists \bar{d})\left(\bar{d} \sqsubseteq^{*} \bar{c} \wedge(\bar{d}, \mathscr{R}) \text { is a witness over } \mathscr{A}_{\gamma}\right) \text { ". }
\end{aligned}
$$

Now we prove that such an iteration exists. We start with the Fréchet filter $\mathscr{A}_{0} \in \mathbf{V}_{0}$. We say " $\left\langle\mathbb{P}_{\gamma}, \mathbb{Q}_{\delta}: \gamma \leq \alpha^{\prime}, \delta<\alpha^{\prime}\right\rangle$ and $\left\langle A_{\gamma}: \gamma \in \alpha^{\prime} \backslash S_{1}^{2}\right\rangle$ and $\left\langle X_{\gamma}: \gamma \in \alpha^{\prime} \cap S_{1}^{2}\right\rangle$ have properties (P1) to (P5)" if all requirements (P1), (P3), and (P4) hold for $\alpha<\alpha^{\prime}$, (P2) holds for $\alpha \leq \alpha^{\prime}$ and (P5) holds for $\beta<\gamma \leq \alpha^{\prime}$.

The following lemma is for the successor steps $\alpha \mapsto \alpha+1$ for $\alpha \notin S_{1}^{2}$.

Lemma 5.1. Assume that $\alpha \in \aleph_{2} \backslash S_{1}^{2}$ and that $\left\langle\mathbb{P}_{\gamma}, \mathbb{Q}_{\delta}: \gamma \leq \alpha, \delta<\alpha\right\rangle$, $\left\langle A_{\gamma}: \gamma \in \alpha \backslash S_{1}^{2}\right\rangle$ and $\left\langle{\underset{\sim}{\gamma}}_{\gamma}: \gamma \in \alpha \cap S_{1}^{2}\right\rangle$ are defined with the properties (P1) to (P5). Then there are some $\mathbb{Q}_{\alpha}, A_{\alpha}$ such that

(a) $\vdash_{\mathbb{P}_{\alpha}} " \mathbb{Q}_{\alpha}$ is proper and preserves $P$-points, so in particular fil( $\left.\mathscr{E}\right) "$,

(b) $\vdash_{\mathbb{P}_{\alpha}} "\left\{\sim_{\alpha}^{A_{\alpha}}\right\} \cup \mathscr{A}_{\alpha}$ is centred",

(c) $\vdash_{\mathbb{P}_{\alpha}}$ "A $\sim_{\alpha}$ is disjoint from infinitely many intervals $\left[\pi_{\alpha}(n), \pi_{\alpha}(n+1)\right)$ ",

(d) property (P5) still holds.

Proof. We let $\mathbb{Q}_{\alpha}$ be the $\mathbb{P}_{\alpha}$-name for Blass-Shelah forcing [6] or $\mathbb{M}$ or Miller forcing or any proper forcing that preserves that $\operatorname{fil}(\mathscr{E})$ is a $P$-point and adds $\left\langle m_{i}: i<\right.$ $\omega\rangle \in{ }^{\omega} \omega$, such that

$$
\left(\forall f \in\left({ }^{\omega} \omega\right)^{\mathbf{V}_{\alpha}}\right)\left(\exists^{\infty} i\right)\left(f\left(m_{i}\right)<m_{i+1}\right) .
$$

Then we let $\underset{\sim}{A}$ be a name for

$$
A_{\alpha}=\bigcup_{i \in \omega}\left[\pi_{\alpha}\left(m_{i}+1\right), \pi_{\alpha}\left(m_{i+1}\right)\right) .
$$

Then claim (c) is true, because $A_{\alpha} \cap \bigcup_{i \in \omega}\left[\pi_{\alpha}\left(m_{i}\right), \pi_{\alpha}\left(m_{i}+1\right)\right)=\emptyset$.

For claim (b) we let $B \in \mathscr{A}_{\alpha}$; actually $B \in[\omega]^{\aleph_{0}} \cap \mathbf{V}_{\alpha}$ is sufficient. We thin out $B$ to $C \in[B]^{\omega}$ that contains at most one point in each interval $\left[\pi_{\alpha}(n), \pi_{\alpha}(n+1)\right)$ and no point in $\left[0, \pi_{\alpha}(2)\right)$. We let $f_{C}: \omega \rightarrow C$ be its increasing enumeration and let $\tilde{f_{C}}$ be the iteration of $f_{C}$, that is, $\tilde{f_{C}}(0)=0, \tilde{f_{C}}(n+1)=f_{C}\left(\tilde{f_{C}}(n)\right)$. Then by 
(5.1) applied to $\tilde{f_{C}} \circ \pi_{\alpha}$, we get

$$
\left(\exists^{\infty} i\right)\left(\tilde{f_{C}}\left(\pi_{\alpha}\left(m_{i}\right)\right) \in C \cap\left[\pi_{\alpha}\left(m_{i}+1\right), m_{i+1}\right) \subseteq\left[\pi_{\alpha}\left(m_{i}+1\right), \pi_{\alpha}\left(m_{i+1}\right)\right)\right),
$$

and hence $A_{\alpha} \cap C$ is infinite.

Now we check property (P5). The only new cases are $\beta \leq \alpha$ and $\gamma=\alpha+1$. By induction hypothesis and by transitivity of $\sqsubseteq^{*}$ we need to consider only $\beta=\alpha$. If $\mathbf{V}_{\alpha}="(\bar{c}, \mathscr{R})$ witnesses over $\mathscr{A}_{\alpha}\left[G_{\alpha}\right]$ ", then $\mathbf{V}_{\alpha+1} \models$ "there is some $\bar{d} \sqsubseteq^{*} \bar{c}$ such that $(\bar{d}, \mathscr{R})$ witnesses over $\mathscr{A}_{\alpha+1}\left[G_{\alpha+1}\right]$ ": We work in $\mathbf{V}_{\alpha}$. Let $\mathscr{R}$ be enumerated as $R_{n}, n \in \omega$. First we thin out $\bar{c}$ to $\left\langle c_{k}^{\prime}: k<\omega\right\rangle$ such that

$$
(\forall k \in \omega)(\forall i \leq k)\left(R_{i}\left(c_{k}^{\prime}\right) \cap \pi_{\alpha}(k+1)=\emptyset\right) .
$$

We let $\bar{c}^{\prime}=\left\langle c_{k}^{\prime}: k<\omega\right\rangle$. In $\mathbf{V}_{\alpha+1}$ let

$$
w=\left\{n<\omega:(\forall k \leq n)\left(R_{k}\left(c_{n}^{\prime}\right) \subseteq A_{\alpha}\right)\right\} .
$$

From $\mathscr{R} \subseteq \mathbf{V}_{\alpha}$ and (5.1), applied to $f(k)=\min \left\{\ell>k+1:(\forall i \leq k)\left(R_{i}\left(c_{k}^{\prime}\right) \subseteq\right.\right.$ $\left.\left.\pi_{\alpha}(\ell)\right)\right\}$, together with equation (5.3), it follows that there are infinitely many $i$ such that $\left(\forall k \leq m_{i}\right)\left(R_{k}\left(c_{m_{i}}^{\prime}\right) \subseteq\left[\pi_{\alpha}\left(m_{i}+1\right), \pi_{\alpha}\left(m_{i+1}\right)\right)\right)$. Since $m_{i}+1<m_{i+1}$, we have that for these $i$, by equation (5.2), $\emptyset \neq\left[\pi_{\alpha}\left(m_{i}+1\right), \pi_{\alpha}\left(m_{i+1}\right)\right) \subseteq A_{\alpha}$, and hence $w$ is infinite.

We let $\bar{d}=\left\langle\bar{c}_{n}^{\prime}: n \in w\right\rangle$. Then $\bar{d} \sqsubseteq^{*} \bar{c}$, and if $C \in \mathscr{\mathscr { A }}_{\alpha+1}\left[G_{\alpha+1}\right]$, then: In the first case, if $C \in \mathscr{A}_{\alpha}\left[G_{\alpha}\right]$, then $(\forall R \in \mathscr{R})\left(R\left(\operatorname{set}(\bar{d}) \subseteq R\left(\operatorname{set}\left(\bar{c}^{\prime}\right)\right) \subseteq \subseteq^{*} C\right)\right.$. In the second case, if $C=A_{\alpha}$, then $(\forall R \in \mathscr{R})\left(R(\operatorname{set}(\bar{d})) \subseteq^{*} C\right)$ by the choice of $w$. Hence we have for all $C \in \operatorname{fil}\left(\left\{A_{\alpha}\right\} \cup \mathscr{A}_{\alpha}\right),(\forall R \in \mathscr{R})\left(R(\operatorname{set}(\bar{d})) \subseteq^{*} C\right)$. Obviously $\mathbb{P}_{\alpha+1}$ preserves $P$-points.

Now we consider two kinds of limit steps, those with countable cofinality and those with cofinalities $\aleph_{1}$ or $\aleph_{2}$. For (P2) we use a well-known preservation theorem: The countable support limit of forcings preserves each $P$-point that is preserved by all approximations [6, Theorem 4.1]. We also use the fact that the countable support limit of proper forcings is proper [18, III, 3.2]. So our iteration preserves $\aleph_{1}$. It preserves $\aleph_{2}$, because any collapse would appear at some intermediate step $\mathbb{P}_{\alpha}$, but $\mathbb{P}_{\alpha}$ has size $\aleph_{1}$ and the $\aleph_{2}$-c.c.

Lemma 5.2. Let $\alpha=\lim _{n} \alpha_{n}$ be the limit of a strictly increasing sequence of ordinals in $\aleph_{2}$. If for each $n,\left\langle\mathbb{P}_{\gamma}, \mathbb{Q}_{\beta}: \beta<\alpha_{n}, \gamma \leq \alpha_{n}\right\rangle$ and the two sequences of names $\left\langle A_{\gamma}: \gamma \in \alpha_{n} \backslash S_{1}^{2}\right\rangle$ and $\left\langle\sim_{\gamma}^{X}: \gamma \in \alpha_{n} \cap S_{1}^{2}\right\rangle$ fulfill (P1) to (P5), then also $\left\langle\mathbb{P}_{\gamma}, \mathbb{Q}_{\alpha}: \beta<\alpha, \gamma \leq \alpha\right\rangle$ and the sequence of names $\left\langle\underset{\sim}{A_{\gamma}}: \gamma \in \alpha \backslash S_{1}^{2}\right\rangle$ and $\left\langle\underset{\sim}{X_{\gamma}}: \gamma \tilde{\in} \alpha \cap S_{1}^{2}\right\rangle$ fulfill (P1) to (P5).

Proof. Again we have to check property (P5) for the new instance $\beta \in \alpha \backslash S_{1}^{2}$ and $\alpha$ itself, given $\beta<\alpha$ and a witness $(\bar{c}, \mathscr{R})$ over $\mathscr{A}_{\beta}$ in $\mathbf{V}_{\beta}$. By induction hypothesis we may possibly increase $\beta$ and assume that $\alpha_{n_{0}}=\beta$ for some $n_{0}$. Then we choose $\bar{c}^{n} \in \mathbf{V}_{\alpha_{n}}, n \in\left[n_{0}, \omega\right)$, in a $\coprod^{*}$-descending manner using (P5) between $\mathbf{V}_{\alpha_{n}}$ and $\mathbf{V}_{\alpha_{n+1}}$, all the time for the same $\mathscr{R}$, and in the end we find some $\bar{d}$ such that for all $n, \bar{d} \sqsubseteq^{*} \bar{c}^{n}$.

Lemma 5.3. Let $\alpha=\lim _{\varepsilon<\kappa} \alpha_{\varepsilon}$ be the limit of a strictly increasing sequence of ordinals in $\omega_{2}$ and let $\kappa$ be $\aleph_{1}$ or $\aleph_{2}$. If for all $\varepsilon,\left\langle\mathbb{P}_{\gamma}, \mathbb{Q}_{\beta}: \beta<\alpha_{\varepsilon}, \gamma \leq \alpha_{\varepsilon}\right\rangle$ and two sequences of names $\left\langle\underset{\sim}{A_{\gamma}}: \gamma \in \alpha_{\varepsilon} \backslash S_{1}^{2}\right\rangle$ and $\left\langle\underset{\sim}{X_{\gamma}}: \gamma \in \alpha_{\varepsilon} \cap S_{1}^{2}\right\rangle$ fulfill (P1) to (P5), then also $\left\langle\mathbb{P}_{\gamma}, \mathbb{Q}_{\beta}: \beta<\alpha, \gamma \leq \alpha\right\rangle$ and the sequences of names $\left\langle A_{\gamma}: \gamma \in \alpha \backslash S_{1}^{2}\right\rangle$ and $\left\langle\underset{\sim}{X_{\gamma}}: \gamma \in \alpha \cap S_{1}^{2}\right\rangle$ fulfill (P1) to (P5). 
Proof. Property (P5) is vacuously true in limit steps. Properties (P3) and (P4) are obviously true.

Finally we carry out the successor step $\alpha \mapsto \alpha+1$ for $\alpha \in S_{1}^{2}$ :

Lemma 5.4. Let $\alpha \in S_{1}^{2}$. Assume that $\left\langle\mathbb{P}_{\gamma}, \mathbb{Q}_{\delta}: \gamma \leq \alpha, \delta<\alpha\right\rangle,\left\langle A_{\gamma}: \gamma \in \alpha \backslash S_{1}^{2}\right\rangle$ and $\left\langle\sim_{\gamma}: \gamma \in \alpha \cap S_{1}^{2}\right\rangle$ fulfill (P1) to (P5) and that the member of the diamond sequence $S_{\alpha}$ is a $\mathbb{P}_{\alpha}$-name for a non-principal ultrafilter $\mathscr{D}$ on $\omega$.

Then there are some $\mathbb{Q}_{\alpha}$ and $\underset{\sim}{X}$, such that $\left\langle\mathbb{P}_{\gamma}, \mathbb{Q}_{\tilde{\alpha}}: \gamma \leq \alpha+1, \delta<\alpha+1\right\rangle$, $\left\langle\underset{\sim}{A_{\gamma}}: \gamma \in(\alpha+1) \backslash S_{1}^{2}\right\rangle$ and $\left\langle\underset{\sim}{X}: \gamma \in(\alpha+1) \cap S_{1}^{2}\right\rangle \tilde{\text { have properties }}$ (P1) to (P5).

Proof. Let $G_{\alpha} \subseteq \mathbb{P}_{\alpha}$ be generic over $\mathbf{V}$ and let $G_{\beta}=\mathbb{P}_{\beta} \cap G_{\alpha}$ for $\beta<\alpha$. We write $\mathscr{A}_{\alpha}$ for $\mathscr{A}_{\alpha}\left[G_{\alpha}\right]$. Let $\left\langle\alpha_{\varepsilon}: \varepsilon<\omega_{1}\right\rangle \in \mathbf{V}$ be increasing continuous with limit $\alpha$, and each $\alpha_{\varepsilon}$ has cofinality $\aleph_{0}$ for $1 \leq \varepsilon<\omega_{1}$ and let $\alpha_{0}=0, \alpha_{1}=\omega+\omega$.

Using Lemma 4.4(1), we can find a sequence $\left\langle\left(\xi_{\varepsilon}, \bar{d}_{\varepsilon}, \mathscr{R}_{\varepsilon}^{\prime}, R_{\varepsilon}^{\prime}, B_{\varepsilon}\right): \varepsilon<\omega_{1}\right\rangle$, such that

(a) for every $\zeta<\omega_{1}$, the sequence $\left\langle\left(\xi_{\varepsilon}, \bar{d}_{\varepsilon}, \mathscr{R}_{\varepsilon}^{\prime}, R_{\varepsilon}^{\prime}, B_{\varepsilon}\right): \varepsilon<\zeta\right\rangle$ belongs to $\mathbf{V}_{\alpha_{\zeta}}$, $\xi_{\varepsilon}<\alpha_{\varepsilon+1}$, and $\operatorname{cf}\left(\xi_{\varepsilon}\right)=\aleph_{0}$,

(b) $R_{\varepsilon}^{\prime} \in\left(\mathscr{R}^{*}\right)^{\mathbf{V}_{\xi_{\varepsilon}}}$,

(c) in $\mathbf{V}_{\xi_{\varepsilon}}$ we have $\bar{d}_{\varepsilon} \in(\mathbb{F})^{\omega}$ and $\mathscr{R}_{\varepsilon}^{\prime} \in\left(\left[\left(\mathscr{R}^{*}\right)\right]^{\aleph_{0}}\right)^{\mathbf{V}_{\xi_{\varepsilon}}}$ and $\left(\bar{d}_{\varepsilon}, \mathscr{R}_{\varepsilon}^{\prime}\right)$ is a witness over $\mathscr{A}_{\xi_{\varepsilon}}\left[G_{\xi_{\varepsilon}}\right]$

(d) $B_{\varepsilon} \subseteq \mathbb{F}$,

(e) every such tuple $\left(\xi_{\varepsilon}, \bar{d}_{\varepsilon}, \mathscr{R}_{\varepsilon}^{\prime}, R_{\varepsilon}^{\prime}, B_{\varepsilon}\right)$ appears in the sequence $\aleph_{1}$ times.

We now choose $\left(\bar{c}_{\varepsilon}, \mathscr{R}_{\varepsilon}\right)$ by induction on $\varepsilon<\omega_{1}$ such that

$(\alpha)\left(\bar{c}_{\varepsilon}, \mathscr{R}_{\varepsilon}\right) \in \mathbf{V}_{\alpha_{\varepsilon}}$ is a witness over $\mathscr{A}_{\alpha_{\varepsilon}}$ and id $\mathrm{id}_{\omega} \in \mathscr{R}_{0}$,

$(\beta)$ if $\zeta<\varepsilon$ then $\bar{c}_{\zeta} \sqsupseteq^{*} \bar{c}_{\varepsilon}$ and $\mathscr{R}_{\zeta} \subseteq \mathscr{R}_{\varepsilon}$,

$(\gamma)$ for all $\zeta<\varepsilon$, if $\left(\bar{d}_{\zeta}, \mathscr{R}_{\zeta}^{\prime}\right)$ witnesses over $\mathscr{A}_{\xi_{\zeta}}$, then there are some $\zeta \leq \zeta^{\prime} \leq \varepsilon$ and some $\bar{c}^{\prime} \sqsupseteq^{*} \bar{c}_{\zeta^{\prime}}$ (the direction is not a mistake) and some $\bar{d}^{\prime} \sqsubseteq^{*} \bar{d}_{\zeta}$ such that $R_{\bar{d}^{\prime}, \bar{c}^{\prime}}=\left\{(m, n):(\exists i)\left(m \in d_{i}^{\prime} \wedge n \in c_{i}^{\prime}\right)\right\}$ and $R_{\bar{d}^{\prime}, \bar{c}^{\prime}} \circ \mathscr{R}_{\zeta}^{\prime} \subseteq \mathscr{R}_{\zeta^{\prime}}$,

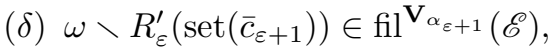

$(\varepsilon) \omega \backslash R_{\varepsilon}^{\prime}\left(\operatorname{set}\left(\bar{c}_{\varepsilon+1}\right)\right) \in \mathscr{D}$,

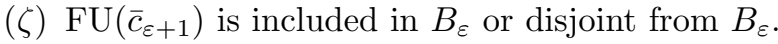

We start the induction with $\alpha_{0}=0, \mathscr{R}_{0}=\left\{\operatorname{id}_{\omega}\right\}$, and we take an arbitrary $\bar{c}_{0} \in(\mathbb{F})^{\omega} . \mathscr{A}_{0}$ is the Fréchet filter.

At limit steps $\varepsilon$ we take the $\bar{c}_{\varepsilon} \sqsubseteq^{*} \bar{c}_{\zeta}$ for all $\zeta<\varepsilon$ and we take $\mathscr{R}_{\varepsilon}=\bigcup_{\zeta<\varepsilon} \mathscr{R}_{\zeta}$. $((\gamma)$ is automatically fulfilled at limit steps. $)$

We carry out the successor step. Suppose $\left(\bar{c}_{\delta}, \mathscr{R}_{\delta}\right), \delta<\varepsilon$, are given and that $\varepsilon$ is countable. We show how to fulfill $(\gamma)$ in successor steps $\varepsilon=\varepsilon^{\prime}+1$ : We enumerate all tasks for item $(\gamma)$ as $\left(\bar{e}^{n}, \hat{\mathscr{R}}^{n}, \zeta_{n}\right), n \in \omega$, and we build $\left(\bar{c}_{\varepsilon^{\prime}}, \mathscr{R}_{\varepsilon}\right)$ by induction in $\omega$ steps as the limit of $\left(\bar{c}_{\varepsilon^{\prime}}, \mathscr{R}^{n}\right)$ that is increasing, actually constant in the first component, in $\left(\leq^{*}, \subseteq\right)$ and witnessing over $\mathscr{A}_{\alpha_{\varepsilon}}$. By the induction hypothesis (P5) below $\alpha$, we may strengthen the $\bar{e}_{\zeta}$ and increase the $\xi_{\zeta}$, and hence we may assume that the $\zeta_{n}$ fulfill $\xi_{\zeta_{n}}=\alpha_{\varepsilon}$ and that $\left(\bar{e}^{n}, \hat{\mathscr{R}}^{n}\right)$ witnesses over $\mathscr{A}_{\alpha_{\varepsilon}}$.

We start with $\mathscr{R}^{-1}=\mathscr{R}_{\varepsilon^{\prime}}$.

We assume that

- $\left(\bar{e}^{n}, \hat{\mathscr{R}}^{n}\right)$ is a witness over $\mathscr{A}_{\alpha_{\varepsilon}}$ (this is the current task for $(\gamma)$ ),

- $\left(\bar{c}_{\varepsilon^{\prime}}, \mathscr{R}^{n-1}\right)$ are already constructed witnessing over $\mathscr{A}_{\alpha_{\varepsilon}}$, and 
- $(\gamma)$ holds for $\left(\bar{d}_{\zeta}, \mathscr{R}_{\zeta}^{\prime}\right)$ that are enumerated among the tasks $\left(\bar{e}^{m}, \hat{\mathscr{R}}^{m}\right), m<$ $n$, with $\hat{\mathscr{R}}^{n-1}$ in the place of $\mathscr{R}_{\zeta^{\prime}}$ and with $\bar{c}^{\prime}=\bar{c}_{\mathcal{\varepsilon}^{\prime}}$ and with $\bar{d}^{\prime}=\bar{e}^{m}$.

Then $R_{\bar{e}^{n}, \bar{c}_{\varepsilon^{\prime}}}^{-1}$ maps $\bar{e}^{n}$ into $\bar{c}_{\varepsilon^{\prime}}$. So $\left(\bar{c}_{\varepsilon^{\prime}},\left\{R_{\bar{e}^{n}, \bar{c}_{\varepsilon^{\prime}}} \circ S: S \in \hat{\mathscr{R}}^{n}\right\}\right)$ witnesses over $\mathscr{A}_{\alpha_{\varepsilon}}$. Hence we may let $\left\{R_{\bar{e}^{n}, \bar{c}_{\varepsilon^{\prime}}} \circ S: S \in \hat{\mathscr{R}}^{n}\right\} \cup \mathscr{R}^{n-1}=\mathscr{R}^{n}$.

In the end we let $\mathscr{R}_{\varepsilon}=\bigcup_{n \in \omega} \mathscr{R}^{n}$. Then $\left(\bar{c}_{\varepsilon^{\prime}}, \mathscr{R}_{\varepsilon}\right)$ witnesses over $\mathscr{A}_{\alpha_{\varepsilon}}$ and the property $(\gamma)$ is carried on. Now we strengthen $\bar{c}_{\varepsilon^{\prime}}$ three times in order to fulfill items $(\delta),(\varepsilon)$, and $(\zeta)$, and we call the outcome $\bar{c}_{\varepsilon}$. For $(\zeta)$ we use the mentioned stronger form of Hindman's Theorem.

Now we let $\mathscr{U}=\operatorname{fil}\left(\left\{\mathrm{FU}\left(\bar{c}_{\varepsilon}\right): \varepsilon<\omega_{1}\right\}\right)$. It is a stable ordered-union ultrafilter by $(\zeta)$ and $(\beta)$. Then we take $\mathbb{Q}_{\alpha}=\mathbb{M}(\mathscr{U})$. It is $\sigma$-centred and hence proper. So (P1) holds.

In $\mathbf{V}_{\alpha}$, the $P$-point $\mathscr{E}$ and the ultrafilter $\mathscr{D}$ are both not Rudin-Blass above $\Phi(\mathscr{U})$, as is secured by $(\delta)$ and $(\varepsilon)$. All potential Rudin-Blass finite-to-one maps are covered by the enumeration $\left\{R_{\varepsilon}^{\prime}: \varepsilon \in \aleph_{1}\right\}=\left(\mathscr{R}^{*}\right)^{\mathbf{V}_{\alpha}}$. By Eisworth's Theorem, Theorem 3.5. the successor $\mathbb{Q}_{\alpha}$ preserves "fil $(\mathscr{E})$ is an ultrafilter". So (P2) holds also for $\mathbb{P}_{\alpha+1}$. Item (P3) is vacuous for $\alpha \in S_{1}^{2}$.

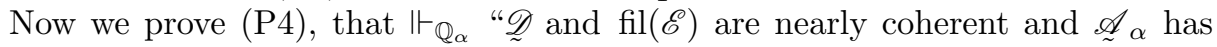
a diagonalisation". First: The near coherence comes from density arguments for $\mathbb{M}(\mathscr{U})$ : Let the generic real $X_{\alpha}=\bigcup\left\{w:(\exists w)\left((w, \bar{c}) \in G_{\alpha+1}\right)\right\}$ be enumerated increasingly by $e_{\alpha}$. Then the generalised inverse of this enumeration $g_{\alpha}(k)=\min \{n$ : $\left.e_{\alpha}(n) \geq k\right\}$ is a finite-to-one function that makes $\mathscr{E}$ and $\mathscr{D}$ nearly coherent: Given $(w, \bar{c}) \in \mathbb{M}(\mathscr{U})$ and $E \in \mathscr{E}$ and $D \in \mathscr{D}$ we get by $(\delta)$ some $E^{\prime} \subseteq E, E^{\prime} \in \mathscr{E}$ and some $\bar{d} \geq{ }^{*} \bar{c}$ such that $E^{\prime}$ avoids $\operatorname{set}(\bar{d})$, and by $(\varepsilon)$ we find some $D^{\prime} \in \mathscr{D}, D^{\prime} \subseteq D$ that avoids set $(\bar{d})$. Now, for two suitable $k<k^{\prime}$, we have $\left[\max \left(d_{k}\right), \min \left(d_{k^{\prime}}\right)\right) \cap D^{\prime} \neq \emptyset$ and $\left[\max \left(d_{k}\right), \min \left(d_{k^{\prime}}\right)\right) \cap E^{\prime} \neq \emptyset$. So $\left(w,\left\langle d_{k}, d_{k^{\prime}}\right\rangle^{\wedge} \bar{d} \mid\left[k^{\prime}+1, \infty\right)\right)$ is stronger than $(w, \bar{c})$ and it forces that $g_{\alpha}(E) \cap g_{\alpha}(D) \neq \emptyset$. Since this works for any two sets, $\mathscr{E}$ and $\mathscr{D}$ are nearly coherent by $g_{\alpha}$.

Second: $X_{\alpha}$ diagonalises $\mathscr{A}_{\alpha}$, since by property $(\alpha),\left(\bar{c}_{\varepsilon}, \mathrm{id}_{\omega}\right)$ is a witness over $\mathscr{A}_{\alpha_{\varepsilon}}$, and this is a complicated way to say that $\operatorname{set}\left(\bar{c}_{\varepsilon}\right)$ is a pseudo-intersection of $\mathscr{A}_{\alpha_{\varepsilon}}$. Since this holds for all $\varepsilon<\omega_{1}$, by genericity $X_{\alpha}$ diagonalises $\mathscr{A}_{\alpha}$.

Next we prove (P5) in the new cases, that is for soe $\beta \in \alpha \backslash S_{1}^{2}$ and for $\alpha+1$. So assume that $\beta<\alpha,(\bar{d}, \mathscr{R})$ is a witness over $\mathscr{A}_{\beta}$ in $\mathbf{V}_{\beta}$ and all later models. For some $\varepsilon_{0}$, we have $\beta<\alpha_{\varepsilon_{0}}$. By $(\gamma)$ we have some $\varepsilon \geq \varepsilon_{0}$ and some $\bar{d}^{\prime} \sqsubseteq^{*} \bar{d}$ and some $\bar{c}^{\prime} \sqsupseteq^{*} \bar{c}_{\varepsilon}$ such that $R_{\bar{d}^{\prime}, \bar{c}^{\prime}} \circ \mathscr{R} \subseteq \mathscr{R}_{\varepsilon}$. Then, by the choice of $\mathscr{U}=$ the filter generated by $\mathrm{FU}\left(\bar{c}_{\delta}\right), \delta<\aleph_{1}$, and of the forcing $\mathbb{M}(\mathscr{U})$, a density argument shows that in $\mathbf{V}_{\alpha+1}$, for all $R \in \mathscr{R}_{\varepsilon}$, the set $R\left(\operatorname{set}\left(\bar{c}_{\varepsilon}\right) \cap X_{\alpha}\right)$ is a pseudo-intersection of $\mathscr{A}_{\alpha}$.

\footnotetext{
${ }^{1}$ Suppose we wanted to simplify (P5) to (P5)': For $\beta<\gamma, \beta, \gamma \in \aleph_{2} \backslash S_{1}^{2}$, if $G_{\gamma} \subseteq \mathbb{P}_{\gamma}$ is generic over $\mathbf{V}$ and $G_{\beta}=\mathbb{P}_{\beta} \cap G_{\gamma}$, then

$$
\begin{aligned}
& \text { if } \mathbf{V}_{\beta} \models " \operatorname{set}(\bar{c}) \text { is a pseudo-intersection of } \mathscr{A}_{\beta} ", \\
& \text { then } \mathbf{V}_{\gamma} \models "(\exists \bar{d})\left(\bar{c} \leq^{*} \bar{d} \wedge \operatorname{set}(\bar{d}) \text { is a pseudo-intersection of } \mathscr{A}_{\gamma}\right) \text { ", }
\end{aligned}
$$

and dispense with the complex requirement $(\gamma)$. Then we would get stuck just at this point here, not knowing how to continue upwards for $(\mathrm{P} 5)^{\prime}$ from an arbitrary $\bar{d}$ with set $(\bar{d})$ being a pseudo-intersection of $\mathscr{A}_{\beta}$. Also, requirements $(\delta)$ to $(\zeta)$ lead to arbitrary $\bar{d}$. So some complexity is necessary, even if at first sight the various items of the list with the Greek letters are not so intertwined and it seems that we carry the original (P5) and $(\gamma)$ with us only for having more technique. Something like (P5) is needed, because otherwise $\mathscr{A}_{\alpha}$ could become nearly ultra for some $\alpha \in S_{1}^{2}$ and by bad luck be in addition nearly coherent to $S_{\alpha}$. Then we could not diagonalise $\mathscr{A}_{\alpha}$ and make $S_{\alpha}$ and fil $(\mathscr{E})$ nearly coherent without destroying $\mathscr{E}$.
} 
So $\left(\bar{c}_{\varepsilon} \cap X_{\alpha}, \mathscr{R}_{\varepsilon}\right)$ witnesses over $\mathscr{A}_{\alpha}$ and since by the definition of the forcing order of $\mathbb{M}(\mathscr{U})$ the set $X_{\alpha}$ splits only finitely many of the $c_{\varepsilon, n}$, that is, there is an infinite set $Y$ such that $X_{\alpha}$ is almost the union over the $c_{\varepsilon, n}, n \in Y$, we get $\bar{c}_{\varepsilon} \cap X_{\alpha} \sqsubseteq^{*} \bar{c}^{\prime}$. Since $R_{\bar{d}^{\prime}, \bar{c}^{\prime}} \circ \mathscr{R} \subseteq \mathscr{R}_{\varepsilon}$, we have $\left(\bar{c}_{\varepsilon} \cap X_{\alpha}, R_{\bar{d}^{\prime}, \bar{c}^{\prime}} \circ \mathscr{R}\right)$ witnesses over $\mathscr{A}_{\alpha}$. Now see: For all $X, R_{\bar{d}^{\prime}, \bar{c}^{\prime}}(X) \supseteq^{*} R_{\bar{d}^{\prime}, \bar{c}_{\varepsilon}}(X)$. So we have that

$$
\left(\bar{c}_{\varepsilon} \cap X_{\alpha}, R_{\bar{d}^{\prime}, \bar{c}_{\varepsilon}} \circ \mathscr{R}\right) \text { witnesses over } \mathscr{A}_{\alpha} \text {. }
$$

We have $R_{\bar{d}^{\prime}, \bar{c}_{\varepsilon}}\left(\bar{d}^{\prime} \cap R_{\bar{c}_{\varepsilon}, \bar{d}^{\prime}}\left(X_{\alpha}\right)\right)={ }^{*} \bar{c}_{\varepsilon} \cap X_{\alpha}$, since $X_{\alpha}$ splits only finitely many $c_{\varepsilon, n}$. We may write $R_{\bar{d}^{\prime} \cap R_{\bar{c}_{\varepsilon}, \bar{d}^{\prime}}\left(X_{\alpha}\right), \bar{c}_{\varepsilon} \cap X_{\alpha}}$ for $R_{\bar{d}^{\prime}, \bar{c}_{\varepsilon}}$ in (5.4), as this is equivalent to it. Hence Lemma 4.4(5) fits literally and we get that $\left(\bar{d}^{\prime} \cap R_{\bar{c}_{\varepsilon}, \bar{d}^{\prime}}\left(X_{\alpha}\right), \mathscr{R}\right)$ is a witness over $\mathscr{A}_{\alpha}=\mathscr{A}_{\alpha+1}$. Of course, $\bar{d}^{\prime} \cap R_{\bar{c}_{\varepsilon}, \bar{d}^{\prime}}\left(X_{\alpha}\right) \sqsubseteq^{*} \bar{d}$.

Now we show that forcing with $\mathbb{P}_{\aleph_{2}}$ gives with the filter $\mathscr{A}$ generated by $\left\{A_{\alpha}\right.$ : $\left.\alpha \in \aleph_{1} \backslash S_{1}^{2}\right\}$ a counterexample to the filter dichotomy principle: If $f(\mathscr{A})$ were ultra, then $f$ would appear in some intermediate step, say in $\mathbb{P}_{\alpha_{0}}$. By known reflection properties of countable support iterations of proper forcings, at an $\aleph_{1^{-}}$ club of later steps $\alpha$ we would have that $f\left(\mathscr{A}_{\alpha}\right)$ is ultra in $\mathbf{V}_{\alpha}$. Hence there is some $\alpha \in S_{1}^{2}$ such that the member $S_{\alpha}$ diamond sequence would guess this ultrafilter $S_{\alpha}=\mathscr{D}=f\left(\mathscr{A}_{\alpha}\right)$. But $X_{\alpha}$ diagonalises $\mathscr{A}_{\alpha}$ and hence $f\left(X_{\alpha}\right)$ diagonalises $f\left(\mathscr{A}_{\alpha}\right)$, and this contradicts the fact that as shown in the proof of Lemma 5.4 there is a finite-to-one $g_{\alpha} \in \mathbf{V}_{\alpha+1}$ coming from the inverse function of the enumeration of $X_{\alpha}$, with $g_{\alpha}\left(f\left(\mathscr{A}_{\alpha}\right)\right)=g_{\alpha}(\mathscr{D})=g_{\alpha}(\mathscr{E})$ being a $P$-point.

The filter $\mathscr{A}$ is not meagre, as $f_{\alpha}\left(A_{\alpha}\right) \neq^{*} \omega$ by (P3), and $f_{\alpha}, \alpha<\aleph_{2}$, enumerates all finite-to-one functions in $\mathbf{V}_{\aleph_{2}}$.

By (P4) and the guessing strength of the diamond, all ultrafilters are nearly coherent to $\mathscr{E}$. So NCF holds in $\mathbf{V}_{\aleph_{2}}$.

So we have proved the Main Theorem.

\section{The Values of SOME CARDinals IN OUR MODElS}

For the definitions of the cardinal characteristics we refer the reader to [1] or [4]. The generic real $r$ added by the Blass-Shelah forcing is not split by any real in the ground model [1, Lemma 7.4.25].

So in the type of models we get when increasing $\mathfrak{s}$ we have $\mathfrak{b}=\mathfrak{u}=\mathfrak{r}=\operatorname{cov}(\mathcal{M})=$ $\operatorname{cov}(\mathcal{N})=\mathfrak{g}=\aleph_{1}$ and $\mathfrak{s}=\operatorname{unif}(\mathcal{M})=\operatorname{unif}(\mathcal{N})=\mathfrak{d}=\aleph_{2}$. This follows from the well-known inequalities in Cichoń's diagramme and from Vojtăś' inequalities that $\mathfrak{r}$ is greater than or equal to both covering numbers [20] and its dual $\mathfrak{s}$ is less than or equal to both uniformities.

However, in the type of models built from Matet iterands $\mathbb{M}$ or Miller iterands in stages $\alpha \notin S_{1}^{2}$, we do not know the splitting number nor the uniformities.

We have in the iteration of $\mathbb{M}$ and $\mathbb{M}(\mathscr{U}) \mathfrak{b}=\mathfrak{u}=\mathfrak{r}=\operatorname{cov}(\mathcal{M})=\operatorname{cov}(\mathcal{N})=$ $\mathfrak{g}=\aleph_{1}$ and $\mathfrak{d}=\aleph_{2}$. From NCF and not FD it follows that in both kinds of models $\mathfrak{u}=\mathfrak{g}=\mathfrak{g}_{f}$ and $\mathfrak{m} \mathfrak{c} \mathfrak{f}=\aleph_{2}$. $\mathfrak{g}_{f}$ is the smallest number of groupwise dense ideals whose intersection is empty and $\mathfrak{m c \mathfrak { f }}$ is the minimal cofinality of the ultrapower $\left(\omega^{\omega}, \leq \mathscr{U}\right)$ for a non-principal ultrafilter $\mathscr{U}$. Note that Brendle constructed a model of $\kappa=\mathfrak{g}<\mathfrak{g}_{f}=\mathfrak{b}=\kappa^{+}$by a c.c.c. forcing [8].

\section{ACKNOWLEDGEMENT}

We thank the referee for a very detailed report and many helpful hints. 


\section{REFERENCES}

1. Tomek Bartoszyński and Haim Judah, Set Theory, On the Structure of the Real Line, A. K. Peters, 1995. MR1350295 (96k:03002)

2. Andreas Blass, Ultrafilters related to Hindman's finite-unions theorem and its extensions, Logic and Combinatorics (S. Simpson, ed.), Contemp. Math., vol. 65, Amer. Math. Soc., 1987, pp. 89-124. MR891244 (88g:04002)

3. - Applications of superperfect forcing and its relatives, Set Theory and its Applications (Juris Steprāns and Steve Watson, eds.), Lecture Notes in Mathematics, vol. 1401, 1989, pp. 18-40. MR1031763 (91b:03081)

4. - Combinatorial cardinal characteristics of the continuum, Handbook of Set Theory (Matthew Foreman, Akihiro Kanamori, and Menachem Magidor, eds.), Kluwer, to appear, available at http://www.math.lsa.umich.edu/ ablass.

5. Andreas Blass and Claude Laflamme, Consistency results about filters and the number of inequivalent growth types, J. Symbolic Logic 54 (1989), 50-56. MR987321 (90a:03076)

6. Andreas Blass and Saharon Shelah, There may be simple $P_{\aleph_{1}}$ - and $P_{\aleph_{2}}$-points and the RudinKeisler ordering may be downward directed, Annals of Pure and Applied Logic 33 (1987), 213-243. MR879489 (88e:03073)

7. _ Near coherence of filters. III. A simplified consistency proof, Notre Dame Journal of Formal Logic 30 (1989), 530-538. MR:1036674 (90m:03087)

8. Jörg Brendle, Distinguishing groupwise density numbers, Monatshefte für Mathematik 152 (2007), no. 3, 207-215. MR2357517

9. Todd Eisworth, Forcing and stable ordered-union ultrafilters, J. Symbolic Logic 67 (2002), 449-464. MR1889561 (2003d:03074)

10. Neil Hindman, Finite sums from sequences within cells of a partition of $N$, J. Combin. Theory Ser. A 17 (1974), 1-11. MR0349574 (50:2067)

11. Jussi Ketonen, On the existence of P-points in the Stone-Cech compactification of integers, Fund. Math. 92 (1976), 91-94. MR0433387 (55:6363)

12. Kenneth Kunen, Set theory, an introduction to independence proofs, North-Holland, 1980. MR.597342 (82f:03001)

13. Pierre Matet, Partitions and filters, J. Symbolic Logic 51 (1986), 12-21. MR830067 (87g:03052)

14. Heike Mildenberger, Groupwise dense families, Arch. Math. Logic 40 (2000), 93-112. MR:1816480 (2003d:03077)

15. Heike Mildenberger, Saharon Shelah, and Boaz Tsaban, Covering the Baire space by families which are not finitely dominating, Annals of Pure and Applied Logic 140 (2006), 60-71. MR2224049 (2007k:03120)

16. Arnold Miller, There are no Q-points in Laver's model for the Borel conjecture, Proc. Amer. Math. Soc. 78 (1980), 103-106. MR.548093 (80h:03071)

17. Andrzej Rosłanowski and Saharon Shelah, Norms on Possibilities I: Forcing with Trees and Creatures, Memoirs of the American Mathematical Society, vol. 141 (no. 671), AMS, 1999. MR.1613600 (2000c:03036)

18. Saharon Shelah, Proper and Improper Forcing, 2nd Edition, Springer, 1998. MR1623206 (98m:03002)

19. Michel Talagrand, Compacts de fonctions mesurables et filtres non mesurables, Studia Mathematicae 67 (1980), 13-43. MR579439 (82e:28009)

20. Peter Vojtáš, Set theoretic characteristics of summability and convergence of series, Commentationes Mathematicae Universitatis Carolinae 28 (1987), 173-184. MR889779 (88i:40001)

Kurt Gödel Research Center for Mathematical Logic, Universität Wien, Währinger Str. 25, 1090 Wien, Austria

E-mail address: heike@logic.univie.ac.at

Institute of Mathematics, The Hebrew University of Jerusalem, Givat Ram, 91904 Jerusalem, Israel - and - Department of Mathematics, Rutgers University, Piscataway, NEW JERSEY 08854

E-mail address: shelah@math.huji.ac.il 